\title{
Leucemia mieloide aguda asociada a tumor de ovario en ausencia de tratamiento antineoplásico previo
}

\author{
Acute myeloid leukemia associated with ovarian tumor in the abscence \\ of prior antineoplastic treatment
}

Sully María Escobar Alberto, * Roxana Martínez Beckerat.**

\section{RESUMEN}

Se presenta caso de adolescente admitida al Hospital Mario Catarino Rivas con historia de fiebre, cefalea y aparición espontánea de hematomas en muslos de 4 días de evolución, acompañado de masa abdominal palpable de $10 \mathrm{~cm}$, con hemograma que reportaba trombocitopenia y anemia. Al realizar Aspirado de médula ósea e inmunofenotipo reportaron Leucemia mieloide Aguda (LMA) estadio M7. La tomagrafía de abdomen reportó extensa tumoración retro peritoneal por lo que se realizó laparotomía exploradora donde se encontró tumor gigante de ovario derecho con metástasis a ganglios paraaórticos. La biopsia reportó un tumor mixto ovárico de células germinales.

La aparición de leucemia mieloide aguda de forma simultánea a tumor de ovario sin antecedente de haber recibido tratamiento quimioterapéutico previo es un hallazgo inusual, y no se encontraron descripciones al respecto en la literatura consultada.

\section{PALABRAS CLAVE}

Células germinativas, Leucemia mieloide, Neoplasias ováricas, Quimioterapia.

\section{ABSTRACT}

This is a clinic report about a teenager, who was admitted to the Mario Catarino Rivas hospital with history of four days of fever, headaches

\footnotetext{
* Médico residente de segundo año del posgrado de Pediatría. Universidad Nacional Autónoma de Honduras, Valle de Sula, UNAH-VS.

* Hematoocóloga Pediatra, Jefa de área de Oncología Pediátrica Hospital Nacional Dr. Mario Catarino Rivas.

Dirigir correspondencia a: sully_sea@hotmail.es

Recibido: 26 de mayo 2015 Aprobado: 14 de junio 2015
}

and spontaneous hematomas on thighs, accompanied by an abdominal mass of $10 \mathrm{~cm}$ of diameter; the hemogram showed anemia and thrombocytopenia. The bone marrow aspirate and immunophenotype reported Acute Myeloid Leukemia (AML) stage M7. The abdominal tomography reported an extense retroperitoneal tumor so an exploratory laparotomy was performed finding a giant right ovarian tumor with metastasis to paraaortic nodes. Biopsy reported a mixed germ cells tumor.

Simultaneous appearence of Acute Myeloid Leukemia and an ovarian tumor without previous history of chemoterapy is an unusual finding that has not been reported in the literature.

\section{KEYWORDS}

Drug Therapy, Germ Cells, Leukemia Myeloid, Ovarian Neoplasms.

\section{INTRODUCCIÓN}

El cáncer es una patología cada vez más frecuente en la población general, incluyendo la infancia. Aunque los pediatras estamos ante la presencia de una aumento de pacientes con cáncer, continúa siendo excepcional la presencia de dos neoplasias con estirpes histológicas diferentes en un mismo momento. Está bien descrita la posibilidad de aparición de una segunda neoplasia, como consecuencia de la terapia establecida para un primer cáncer. Tal es el caso de tumores que aparecen en áreas previamente radiadas, o la aparición de Leucemias Mieloides secundarias al uso de agentes alquilantes o epipodofilotoxinas. 
La leucemia es el cáncer más frecuente en la edad pediátrica, con un claro predominio de las linfoblásticas. Las leucemias mieloides corresponden a un $20 \%$ del total de leucemias. $^{(1)}$

Los tumores malignos de ovario son raros en niñas. Son neoplasias de crecimiento rápido que surgen de las células germinales primordiales derivadas de las gónadas embrionarias, alcanzando dimensiones impresionantes en periodos cortos de tiempo. Ocurren en un 2.9\% del total de neoplasias en niñas y adolescentes. ${ }^{(2,3)}$

\section{REPORTE DE CASO}

Paciente femenina de 13 años de edad con historia de fiebre subjetivamente alta, no cuantificada, sin predominio de horario de 4 días de evolución acompañado de cefalea frontal, pérdida del apetito, debilidad generalizada, con hematomas espontáneos en ambos mus-los, anemia y trombocitopenia. Los resultados laboratoriales se muestran en la Tabla No. 1.

En su evaluación inicial; pálida, crónicamente enferma, con hematomas en muslos y piernas, con masa de bordes lisos, no móvil en hemiabdomen derecho, que alcanzaba $10 \mathrm{cms}$ bajo el reborde costal. Aunque esta masa no fue el motivo de consulta, la madre refirió haber notado la distención progresiva del abdomen en las últimas semanas, pero no le alarmó pues no le causaba dolor. Al caminar fue notable un defecto en su marcha por una deformidad en genu varo, pero no existían otras anormalidades fenotípicas que llamaran la atención u orientaran a un síndrome congénito. No se encontraron adenopatías ní visceromegalias.

Con estos hallazgos se presentó al servicio de hematoncológia pediátrica, y se sometió a aspirado de médula ósea y toma de muestra para inmunofenotipo.
Tabla No. 1: Principales resultados laboratoriales de paciente

\begin{tabular}{lc}
\hline Exámen & Resultado \\
\hline Leucocitos & 16,500 \\
Neutrófilos & $70 \%$ \\
Linfocitos & $25 \%$. \\
Hemoglobina & $6.3 \mathrm{~g} / \mathrm{dl}$ \\
Plaquetas & $6 \times 10 \% / \mathrm{L}$ \\
Lactato deshidrogenasa & 1,406 \\
AFP * inicial $\quad>300 \mathrm{ng} / \mathrm{L}(\mathrm{norm} .:<10 \mathrm{ng} / \mathrm{ml})$ \\
AFP* posquimioterapia & $254 \mathrm{ng} / \mathrm{L}$ \\
Urocultivo & Negativo \\
Hemocultivo & Negativo \\
LDH $^{* *}$ & $1,406 \mathrm{ul} / \mathrm{l}(100 \mathrm{a} 333 \mathrm{ul} / \mathrm{l})$ \\
\hline
\end{tabular}

*AFP: Alfafetoproteina, ${ }^{*}$ LDH: lactato deshidrogenasa.

Se realizó tomografía abdominal (Fig. 1 y 2) que mostró extensa tumoración retroperitoneal que se extendía desde L1 hasta hueco pélvico impresiona corresponder con ovario derecho.

Se interconsultó con el servicio de Cirugía Pediátrica quienes consideraron indispensable realizar laparotomía exploradora pero por las alteraciones hematológicas con que cursaba la paciente el procedimiento fue referido de forma electiva hasta estabilizar la adolescente.

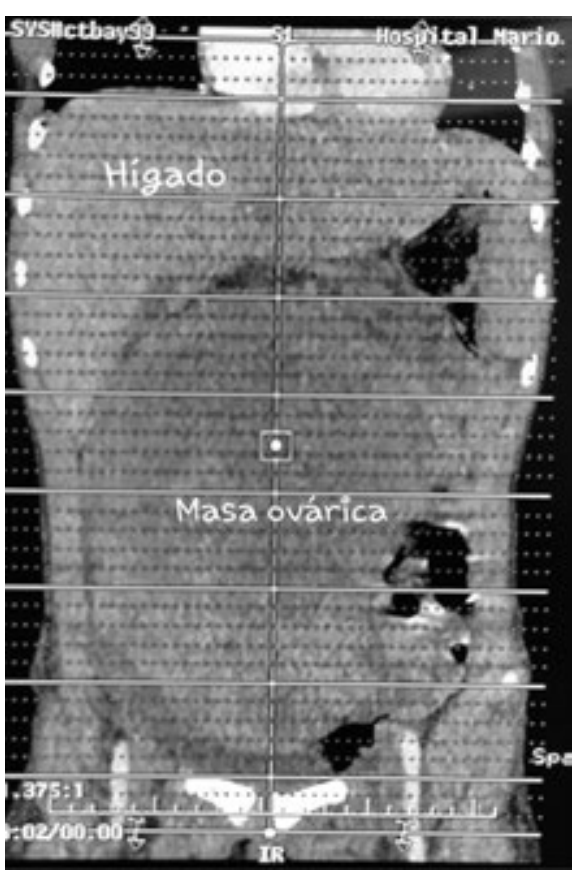

Figura No. 1: TAC abdomen muestra masa ovárica de L1 hasta hueco pélvico 


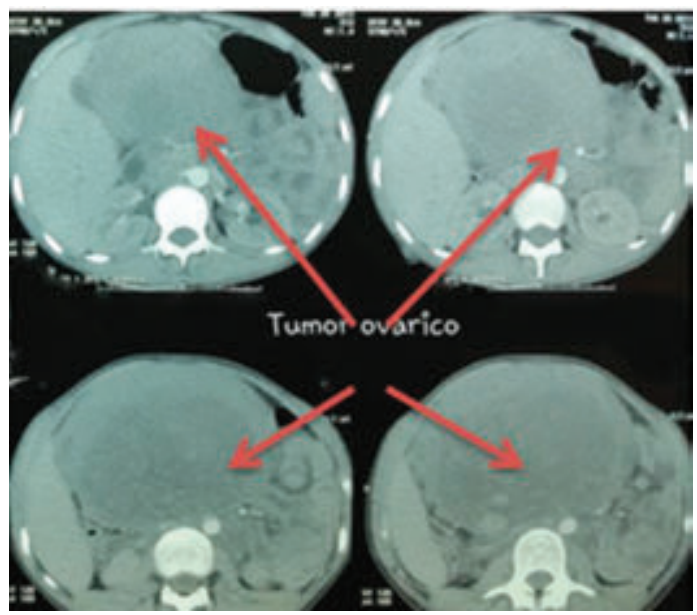

Figura No. 2: TAC abdomen Corte transversal muestra tumor ovario derecho

La paciente inició terapia de inducción para LMA de acuerdo al protocolo de la Asociación de Hemato Oncología Pediátrica Centroamericana (AHOPCA) 2007. Permaneció ingresada por diferentes complicaciones hematológicas e infecciosas. Al día 28 del inicio de quimioterapia, ya resuelta la pancitopenia postquimioterapia, la paciente fue sometida a laparotomía exploradora encontrando tumor gigante $(20 \mathrm{x}$ $15 \mathrm{~cm}$ ) (Fig. 3) dependiente de ovario derecho. Sus características; tumor blanquecino, sólido, con líquido peritoneal cetrino. En el transoperatorio se evidenció que también había compromiso del ovario izquierdo; consistencia dura, y con tumoración que sobresalía de la cápsula y el ligamento tuboovárico. Se practica salpingoooforectomia bilateral y omentectomía con biopsia de ganglio mesentérico. Se preservó útero por estar aparentemente sano, y por petición de la familia.

Patología definió que la masa correspondía a tumor mixto de células germinales en ambos ovarios. Líquido peritoneal positivo por malignidad. Epiplón se reporta con vasos congestivos sin evidencia de ganglios. Muestra de ganglio mesénterico reportó que contenía nódulo de $1 \mathrm{~cm}$ y ganglio paraaórtico con nódulo de $2 \mathrm{~cm}$.

La paciente evolucionó satisfactoriamente en el postoperatorio. Fué egresada tres días después de la cirugía, y 15 días del inicio de la quimioterapia. En su reingreso un mes después recibiendo su quimioterapia la paciente falleció.

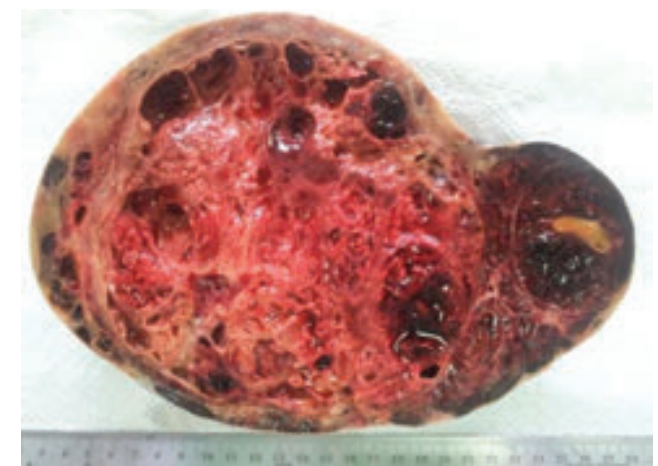

Figura No. 3: Corte Macroscópico biopsia de ovario. Tumor mixto de células germinales extraído de paciente de13 años.

\section{Discusión}

Encontrar tumoraciones en más de un sitio es frecuente en pacientes con cáncer en todas las edades. Normalmente, lesiones histológicamente similares en órganos diferentes corresponden a metástasis y es rutinario buscarlas en determinados sitios, de acuerdo al órgano primario. Así, en osteosarcoma buscamos siempre la posibilidad de metástasis pulmonares, por ejemplo.

Otras lesiones pueden aparecer en órganos pares, ya sea de manera simultánea (sincrónicos) o no (metacrónicos), como ocurre en el retinoblastoma, donde pueden ocurrir dos tumores primarios en ambos ojos, debido a alteraciones cromosómicas constitucionales predisponentes. Otro caso bien descrito es el de los síndromes de Neoplasia Endócrina múltiple.

Los tumores primarios múltiples se definen como la coexistencia de más de una neoplasia primaria en diferentes órganos, o coexistencia de dos o más neoplasias primarias de diferentes tipos celulares en el mismo órgano. Excluyendo todas las neoplasias multifocales en el mismo órgano (tumores de vejiga), las neopla- 
sias simultáneas en el mismo órgano o en órganos pares (cáncer de mama), neoplasias asincrónicas en el mismo órgano o en órganos pares (pólipos de colon) y enfermedad neoplásica progresiva (carcinoma cervical in situ $y$, subsiguientemente, carcinoma de cérvix). ${ }^{(3)}$

Esta definición claramente excluye a las segundas neoplasias, que se definen como Crecimientos anormales de tejido que siguen a una neoplasia previa pero que no son una metástasis de ésta. La segunda neoplasia puede tener el mismo o diferente tipo histológico y puede ocurrir en los mismos o diferentes órganos que la neoplasia previa pero en todos los casos surgen a partir de un evento oncogénico independiente. El desarrollo de la segunda neoplasia puede o no relacionarse con el tratamiento para la neoplasia prévia ya que el riesgo genético o los factores predisponentes pueden ser en realidad la causa. ${ }^{(3)}$ El caso reportado en este manuscrito, es muy llamativo por presentar en realidad tres neoplasias: dos de ellas afectando a ambos ovarios, y la tercera de estirpe hematopoyética, una Leucemia Mieloide aguda M7 por FAB.

Los tumores ováricos son raros en la edad pediátrica y representan del 1 al $5 \%$ de los tumores infantiles. Son más frecuentes entre los 9 y 12 años $^{(1-5)}$ y alcanzan mayor porcentaje de malignidad en las niñas de mayor de edad. ${ }^{(2,}$ 6,7) El tipo histológico más frecuente es el teratoma, con claro predominio de su variante quística o dermoide, que representa el $40-75 \%$ de los tumores ováricos. $\left.{ }^{(3,} 7,8\right)$ En este caso, patología reportó tumor mixto de células germinales en ambos ovarios.

Es habitual determinar los niveles de marcadores tumorales de línea germinal como alfafetoproteína y subunidad beta de la gonadotrofina coriónica, que resultarán elevados en muchos, aunque no todos los pacientes. En el caso de nuestra paciente los marcadores alfafetoproteina preoperatoria era de $300 \mathrm{ng} / \mathrm{ml}$ y la postoperatoria de $256 \mathrm{ng} / \mathrm{ml}$ elevadas según el valor normal que debe ser inferior a $15 \mathrm{ng} / \mathrm{ml}$. ${ }^{(9)}$
La presencia de dos tumores ováricos simultáneos de estirpe germinal en la infancia está descrita en casos de disgenesia gonadal. El síndrome de Swyer por ejemplo es una disgenesia gonadal pura, con cariotipo 46XY, fenotipo femenino normal, y ausencia completa de tejido gonadal funcional, que está representado por unas cintillas gonadales rudimentarias, bilaterales, no funcionantes, compuestas por tejido fibroso, en presencia de genitales internos femeninos normales. En un 20\% existe mutación o deleción del gen SRY. En el $80 \%$, el SRY es aparentemente normal. El riesgo de neoplasia gonadal es alto; está indicado practicar gonadectomía profiláctica. El gonadoblastoma y el disgerminoma son las neoplasias más comúnmente asociadas. ${ }^{(10)}$ En el Turner las principales manifestaciones clínicas son relacionadas con alteraciones linfáticas (cuello ancho con piel abundante, linfoedema, displasia de uñas y alteraciones en los dermatoglifos). Las relacionadas con trastornos del esqueleto incluyen talla baja, cuello corto, micrognatia, cubitus valgus, metacarpianos y metatarsianos cortos, genu varo. Otros órganos afectados pueden incluir corazón y riñones, y anomalías cráneo-faciales características. Respecto a nuestra paciente, esta se encontraba en el percentil 3 para su edad, y tenía Genu-Varo marcado que dificultaba su marcha, sin otras malformaciones congénitas aparentes.

Lo particular de este caso es que presentaba también una segunda neoplasia, concomitante, pero de estirpe histológica diferente. Morfológicamente correspondía a una LMA M7 (Leucemia megacarioblástica). El inmunofenotipo presentaba CD61 38.86\%, CD42b 64.13\% y CD36 24.57\%. En este caso no se realizó biopsia de médula ósea, pero típicamente presentan algún grado de mielofibrosis, que también es característica de la enfermedad. La LMA M7 constituye entre el 4 y $15 \%$ de todas las leucemias mieloides, pero es 500 veces más común en pacientes con síndrome de Down, y la desarrollarán todos aquellos que han presentado síndrome mieloproliferativo transitorio del 
recién nacido. Paradójicamente, el pronóstico es mucho mejor entre niños con trisomía 21 que en el resto de pacientes. ${ }^{(11)}$ También la M7 está asociada a la translocación ${ }^{(1,22)}$ en pacientes lactantes, pero no en niños mayores. Esta translocación también confiere buen pronóstico.

En los protocolos americanos la tasa de sobrevida con quimioterapia intensiva y trasplante es de 35\% para pacientes sin Síndrome de Down (SD), pero superior al $75 \%$ para niños con el síndrome. ${ }^{(12)}$ La paciente inició terapia de inducción para LMA de acuerdo al protocolo de la Asociación de Hemato Oncología Pediátrica Centroamericana (AHOPCA) 2007, sin embargo debido a lo complicado de su caso y la asociación con tumores de ovario, la paciente falleció.

También está implicada en al M7 la mutación GATA-1, que se supone ocurre en útero en los niños con Síndrome de Down que presentan la enfermedad en el período neonatal. ${ }^{(13)}$ Las Leucemias megacarioblásticas tienen un pronóstico inferior al resto de las Leucemias mieloides agudas. Existe un tercer grupo de M7: las que son secundarias a terapia para leucemias linfoblásticas, y que han recibido derivados de la topoisomerasa, que es una causa conocida para a M7. ${ }^{(14-16)}$

Lastimosamente los recursos locales no nos permitieron definir con claridad la presencia de mutaciones constitucionales en esta paciente, ni de mutaciones específicas en las células tumorales de ovario y de médula ósea. Sin embargo, es probable que una misma mutación o algún rearreglo genético pudieran estar causando estas dos neoplasias, ya que es excepcional que surjan dos neoplasias de estirpes tan diferentes.
Estudios efectuados en casos de localización ovárica arrojan resultados de supervivencia del $75 \%$ con PEB (cisplatino, etopósido, bleomicina), 55\% con PVB y 63\% con VAC (vincristina, actinomicina D, ciclofosfamida). El Children's Oncology Group (COG) concluye en una reciente publicación que los tumores germinales ováricos tienen un excelente pronóstico con tasas de supervivencia a 6 años que oscilan del $95 \%$ al $93 \%$ en los estadios I a IV respectivamente, cuando se utilizan protocolos que contengan cisplatino, y que la cirugía conservadora es adecuada.(5) Lastimosamente como comentamos antes, la M7 tiene un pronóstico muy pobre en pacientes sin SD, y en estos pacientes el pronóstico depende del logro de remisión en la primera inducción, y de la posibilidad de contar con un donante de médula ósea para lograr un trasplante, que constituye actualmente el tratamiento de elección posterior al logro de la remisión.

En conclusión, nos encontramos ante un caso excepcional, en una paciente femenina, adolescente, con malformaciones constitucionales menores, sin diagnóstico sindrómico y sin antecedentes patológicos antes de este ingreso, que se ve obligada a afrontar tratamiento antineoplásico con quimioterapia sumamente intensiva para una leucemia de muy mal pronóstico, y con fallo en el logro de la remisión. En cuanto a sus otros tumores, solo pudo ofrecerse tratamiento quirúrgico, puesto que no podía recibir de manera simultánea dos esquemas diferentes de tratamiento. Lastimosamente, la enfermedad fue rápidamente progresiva durante su segunda inducción y falleció a consecuencia de ello. 


\section{BIBLIOGRAFÍA}

1. Instituto Nacional del Cáncer NIH [internet]. Estados Unidos de América: Leucemia mieloide aguda y otras neoplasias mieloides malignas infantiles: Tratamiento (PDQ ${ }^{\circledast}$ ). [Actualizado 17 abril 2015. Consultado el 25 de mayo 2015].Disponible en: http://www.medigraphic.com/pdfs/circir/ cc-2007/cc072e.pdf.

2. Quero A, Estrada R, Tenorio H. Tumor de células germinales de ovario: características clínicas y resultado de tratamiento. Rev. Mex.Cirugía y cirujanos [Revista en internet]. 2007 [citado 25 de mayo 2015];75 (2):81-85. http://www.redalyc.org/articulo0 a?id=66275205.

3. Coquard Isabelle. Ovarian germ cell malignant tumors. Orphanet Encyclopedia. Francia [actualizado Marzo 2004; consultado el 28 mayo 2015]. Disponible en: https:// www.orpha.net/data/patho/GB/uk-OVA Rl.pdf.

4. Vachany C. Guía del paciente a los marcadores tumorales. The Abramson Cancer Center of the University of Pennsylvania. [Actualizado 6 agosto 2013. Consultado el 5 de mayo 2015]. Disponible en: http://es.oncolink.org/treatment/article. $\mathrm{cfm}$ ?aid=560\&id=296.

5. Pardo GN, Muñoz VA, Maldonado RS. Tumores de células germinales. Clin Transl Oncol. Elsevier [revista en internet]. 2005 [citado 10 mayo 2015] 7(8):361-9. Disponible en: http://apps.elsevier.es/watermark/ctl _servlet?_f=10\&pident_articulo=1307893 $3 \&$ pident_usuario $=0 \&$ pcontactid $=\&$ pi den t_re vista $=57 \&$ ty $=169$ \&accion $=$ L\& origen $=$ zona delectura\&web $=w w w$.elsevier.es\&lan $=e s \&$ fichero $=57 \mathrm{v} 07 \mathrm{n} 08 \mathrm{a} 13078933 \mathrm{pdf}$ 001.pdf.
6. Heras AMM, Gelabert MA. Tumores primarios múltiples independientes y segundas neoplasias primarias: Relación con el hábito de fumar. Actas Urol Esp [revista en internet]. 2010 [citado 2015 Jun 2 ]; 34(6): 516-521 Disponible en: http://scielo.isciii. es/pdf/aue/v34n6/especial2.pdf.

7. Díaz R, Aparicio J. Leucemias agudas y Síndromes mielodisplásicos secundarios al tratamiento oncológico. En Anales de medicina interna [revista en internet] 2003 Mayo [citado 2015 May 20]; 20 (5):257-268. Disponible en: http://scielo.isciii.es/scielo. php? pid=S0212-71992003000500009\&s cript=sci_arttext.

8. Instituto Nacional del Cáncer NIH [internet]. Estados Unidos de América: Tumores de Células germinativas de ovario: tratamiento $\left(\mathrm{PDQ}^{\oplus}\right)$. [Actualizado 5 marzo 2015. Consultado el 25 de mayo 2015].Disponible en: http://www.cancer.gov/espanol/tipos/ova rio/pro/tratamiento-celulas-germinati vas-ovario-pdq.

9. Health link Children's oncology group [internet]. Estados Unidos de América. Viviendo saludable después del tratamiento para el cáncer infantil. Actualizado en el 2013.consultado 15 de mayo 2015]. Disponible en: http://survivorshipguideli nes.or $\mathrm{g} / \mathrm{pdf} /$ IntroductiontoLong-TermFo llow-Up \%20-\%20Spanish.pdf.

10. Gonzalo Alonso E, Merino Marcos I, Fdez-Teijeiro Alvarez A, Astigarraga Aguirre I, Navajas Gutiérrez A. Tumores ováricos en la infancia: a propósito de una revisión casuística. Anales Esp Pediatr. [revista en internet] 1998 Sept [citado 10 May 2015]; 49 (5): 491-494. Disponible en: http:// www.aeped.es/sites/default/files/anales/ 49-5-11.pdf. 
11. Salazar Al, Palomeque F, Pascual E, Porras V. Disgerminoma y síndrome de Swyer. Progresos de Obstetricia y Ginecología [revista de internet]. 2006 [citado 1 May 2015]; 49(3):154-158. Disponible en: http:// linkinghub.elsevier.com/retrieve/pii/ S0304501306725869?via=sd.

12. Athale UH, Razzouk BI, Raimondi SC, Xin Tong, Behm FG, Head DR, et. al. Biology and outcome of childhood acute megakaryoblastic leukemia: a single institution's experience. Blood [revista en internet]. 2001 [citado 2 junio 2015]; 97(12):3727-3732. Disponible en: http://www.bloodjournal. org/content/97/12/3727.full-text.pdf+ht $\mathrm{ml}$.

13. Barnard DR, Alonzo TA, Gerbing RB, Beverly Lange, Woods WG. Comparison of childhood myelodysplastic syndrome, AML FAB M6 or M7, CCG 2891: report from the Children's Oncology Group. Pediatric blood \& cáncer [revista en internet]. 2007 [citado el 6 de junio 2015]; 49(1):17-22. Disponible en: http://onlinelibrary.wiley.com/doi/10. 1002/pbc.20951/abstract?deniedAccess CustomisedMessage $=\& u s e$ rlsAuthenticated $=$ false.
14. Druker BJ, Sawyers Ch L, Hagop Kantarjian, Resta DJ, Fernandes Reese S, Ford JM, et al. Activity of a specific inhibitor of the $B C R-A B L$ tyrosine kinase in the blast crisis of chronic myeloid leukemia and acute lymphoblastic leukemia with the Philadelphia chromosome. New England Journal of Medicine [revista de internet]. 2001 [citado el 6 Junio 2015].344(14):1038-1042.

Disponible en: http://www.nejm.org/doi/ full/10.1056/NEJM200104053441402.

15. Canto $P$, Kofman-Alfaro $S$, Jiménez $A L$, Söderlund D, Barrón C, Reyes E, et al. Gonadoblastoma in Turner syndrome patients with nonmosaic 45, $X$ karyotype and $Y$ chromosome sequences. Cancer genetics and cytogenetics [revista en internet]. 2004 [citado 8 Jun 2015]; 150 (1):70-72. Disponible en: http://www.can cergeneticsjournal.org/article/S0165-4608(03)00345-5/ abstract.

16. Rutgers JL, Scully RE. Pathology of the testis in intersex syndromes. En Seminars in diagnostic pathology. 1987. p. 275-291. 